\title{
Thinking about Availability in Large Service Infrastructures
}

\author{
Jeffrey C. Mogul, Rebecca Isaacs, and Brent Welch - Google Inc., Mountain View, CA
}

ACM Reference format:

Jeffrey C. Mogul, Rebecca Isaacs, and Brent Welch - Google Inc., Mountain View, CA. 2017. Thinking about Availability in Large Service Infrastructures. In Proceedings of HotOS '17, Whistler, BC, Canada, May 08-10, 2017, 6 pages.

https://doi.org/10.1145/3102980.3102983

\section{Introduction}

With the rise of the Internet, the Web, and cloud computing, we have come to depend on a complex stack of interdependent online services. Successful operation of a cloud-hosted service, such as NetFlix or SnapChat, can depend on dozens of underlying distributed systems, some of which in turn depend on each other. While most people (excepting teenagers) do not view SnapChat as a life-critical service, past failures of systems such as Healthcare.gov [12, 33] have had real-world consequences. Cloud providers are under pressure to deliver more "nines" of availability as traditional enterprise computing moves to the cloud.

The need for an end-to-end approach to availability is highlighted by the "blameless postmortem" culture of some providers [5, Ch. 15]. However, learning the patterns of successful and harmful practices does not in itself lead to the creation of principles that can defend against the unexpected. Meanwhile, the research community has not given the same attention to these end-to-end availability issues, especially for large-scale infrastructures, as it has to various point solutions, such as distributed consensus and state-machine replication (see $\S 5$ ).

We have observed that system designers struggle to define overall availability goals suitable for large infrastructures, and then struggle again to convert these to goals for component services. In this paper, we describe the challenges of providing a precise and principled definition for availability ( $\$ 3)$, the possibility of thinking about availability in much the same way that we have learned to think about security ( $(4)$, and some general ideas for designing highly-available infrastructures $(\S 6)$. We do not claim to have solved these challenges.

\subsection{Context}

Our company has learned to design and operate planetaryscale services with reasonably high availability. Historically, these have been Software as a Service (SaaS) systems (search, YouTube, GMail, etc.), implemented as scale-out distributed systems that could tolerate all sorts of failures in lower layers, through the use of traditional techniques such as replication, distributed consensus algorithms (e.g. Paxos), and transactions,

Permission to make digital or hard copies of part or all of this work for personal or classroom use is granted without fee provided that copies are not made or distributed for profit or commercial advantage and that copies bear this notice and the full citation on the first page. Copyrights for third-party components of this work must be honored. For all other uses, contact the owner/author(s).

HotOS '17, May 08-10, 2017, Whistler, BC, Canada

(C) 2017 Copyright held by the owner/author(s)

ACM ISBN 978-1-4503-5068-6/17/05.

https://doi.org/10.1145/3102980.3102983 or newer techniques like straggler-tolerance [17]. Our underlying infrastructure is based on a large number of relatively small compute and storage nodes, and on networks built from large numbers of small switches [39]. So, as long as low-level faults are uncorrelated, the overall SaaS systems keep running.

Two trends have driven us to improve the availability of our infrastructure, while continuing to exploit the cost advantages of relatively unreliable components. First, users who originally adopted low-cost SaaS services as useful novelties now increasingly rely on these services. (E.g., automobile drivers have shifted from paper maps, to GPS devices with self-contained maps, to online apps with real-time traffic reports.)

Second, and more challenging, the growth of Infrastructureas-a-Service (IaaS) cloud-computing platforms makes availability of the underlying infrastructure significantly more critical, since many IaaS tenants lack the expertise or scale to build applications that tolerate low-level faults ${ }^{1}$, yet desire the low costs associated with cheap scale-out hardware.

Prior work by colleagues [21] categorizes and analyzes a large data set of network failures, and describes techniques for improving availability. Here we focus on broader aspects of availability, and we look at distributed systems in general.

\section{A simple case study}

In parts of this paper, we will use an existing, moderately complex stack of services, depicted in Fig. 1, as an example to make our discussions more concrete.

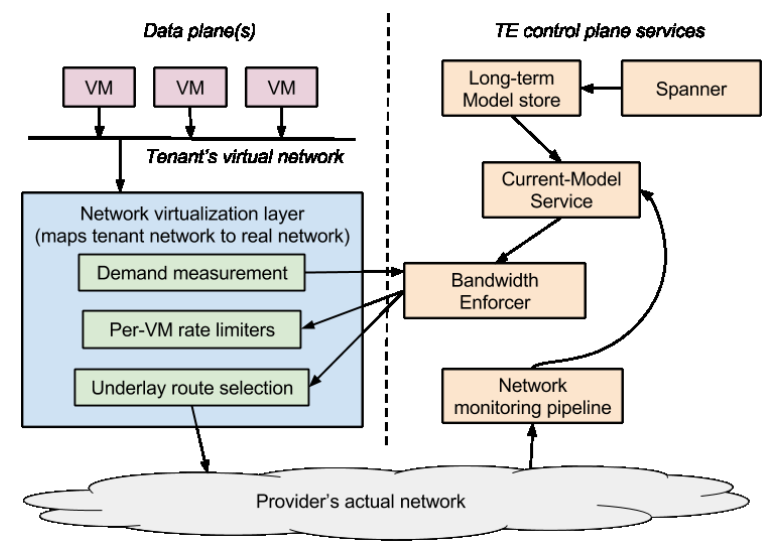

Figure 1: Traffic engineering (TE) infrastructure

Consider a cloud VM that wishes to communicate over the network. To meet its bandwidth needs while isolating it from other tenants, we route its packets over less-utilized links, while throttling its maximum rate; these are two aspects of traffic engineering (TE), which we implement using Bandwidth Enforcer (BwE) [29]. BwE in turn depends on a Current-Model Service, which builds an abstract model of the network's current state (link utilization and link failures). That service uses

${ }^{1}$ including "legacy" applications that must be ported to IaaS platforms without significant rewrites. 
low-level network monitoring data delivered by a scalable network monitoring pipeline, as well as static models of the network's intended state. Models reside in a Long-term Model Store, using Spanner [16] for consistent storage.

\section{Defining infrastructural availability}

Without a precise definition of infrastructural availability, it is impossible to evaluate whether availability goals are being met, yet we continually struggle to find such a definition. We believe this confusion results from (1) the underlying multidimensionality of a precise definition, (2) the need to reduce this dimensionality to something that is feasible to measure and explain, and (3) the need to then decompose a top-level goal into goals for the component services.

Defining availability starts with choosing Service Level Indicators (SLIs): something feasibly measurable about the utility of a system, like latency, packet loss or network path length. A simple Service Level Objective (SLO) is just a tuple: (SLI, comparator, threshold) [5, Ch. 4]. In reality, meaningful SLOs are multi-dimensional, which means that we must define a function over multiple SLIs that says whether the system is meeting its objectives. Much of the confusion around availability definitions involves choosing this composition function, as well as the underlying SLIs. ${ }^{2}$

\subsection{Reasons for multi-dimensional SLOs}

What makes real-world availability SLOs multidimensional? We discuss several dimensions below (there are others!)

Probabilistic SLOs: We generally cannot afford to build systems that truly never fail, so SLOs are often probabilistic in several dimensions. An SLO of " $99.9 \%$ of packets will arrive with low delay, during $99.5 \%$ of the minutes during a month" allows for some minor short-term congestion and some rare switch reboots, but complicates SLO definition by requiring us to choose a second threshold and several measurement intervals.

Performance: Performance and availability are often treated as orthogonal. For example, Brewer writes that "the CAP theorem ignores latency, although in practice, latency and partitions are deeply related". A system that never times out its internal communication is placing consistency over availability [7]. However, for a tenant whose VMs can communicate but only with inadequate bandwidth, its virtual network is "unavailable" for practical purposes.

Therefore, useful availability SLOs are best defined over performance SLIs; e.g., "the service handles 1000 requests/sec at a $99 \%$ ile latency < 100msec. during $99.99 \%$ of the minutes in a month." (Note that even a performance-only SLO can be multi-dimensional, requiring a minimal throughput at a maximum latency.)

There are numerous public examples that demonstrate a lack of integration between the measurement of performance and availability. The root cause of a major AWS outage was described as a complicated inter-dependency between a brief network disruption and service load. AWS proposed "stricter monitoring on performance dimensions" as one defense [3].

\footnotetext{
${ }^{2}$ Contracts between providers and customers are stated as Service Level Agreements (SLAs); an SLA is an "SLO with consequences" (such as a substantial refund) [5, Ch. 4], [44]. Providers sometimes use SLAs for internal interfaces, as well, to allocate blame or bonuses. SLA definition, inherently involving legal, managerial, or marketing concerns, is beyond our scope.
}

Multiple kinds of operation: Systems are often required to have different availability guarantees for different types of operation, e.g., reads vs writes, sends vs receives. Thus, we may wish to define SLIs for each individually; e.g., travellers can check flight status even if they cannot update reservations (favoring reads over writes), while security logging systems value uninterrupted appends over read availability.

We may also need separate SLOs for control-plane and dataplane availability (e.g., existing VMs should keep running even when we cannot create new ones). Complex control-plane APIs might provide higher availability for local operations (create a new VM) than for global ones (create an administrator account).

Fig. 1 helps to illustrate these points. The VMs should be able to communicate, perhaps with degradation that increases over time, even if BwE is down (data-plane availability > control-plane availability). BwE can continue to throttle based on new demand measurements even if the Current Model Store is down, but with less accuracy (read-stale > read-consistent).

\subsection{Dimensionality reduction}

The second source of difficulty in defining availability is the need for dimensionality reduction. In practice, nobody uses SLOs defined via all relevant dimensions; they are too hard to understand, and measuring all the SLIs costs too much. So, we need a way to reduce this complexity, either by using proxy SLIs (e.g., packet-loss rates as a proxy for TCP throughput), or by constructing weighted sums over SLIs. Much of the debate we observe is over how to balance utility with feasibility in this dimensionality reduction.

One common approach is to define tiered SLOs, where you pay more for a better threshold, or set thereof. (These are often called "gold," "silver," and "bronze" SLOs.) This shifts some control over dimensionality reduction to customers, but also shifts conceptual complexity, which can create confusing choices [15]. Another is to sweep the complexity under the rug: Azure user documentation [20] alludes to "effective availability" as the cumulative effect on total system availability of all the constituent service SLAs, but the SLAs do not cover performance, and the advice to users is simply to "take care."

\subsection{Choosing sub-system SLOs}

After choosing top-level SLOs, one must then decompose this into individual SLOs for component sub-systems. The relationship between component and end-to-end SLOs is nontrivial; naive approaches (such as multiplying probabilities or subdividing downtime budgets) can mislead in both directions: - Good choices for subsystem SLOs depend on an accurate understanding of dependencies between components in a complex, multi-layered infrastructure; unknown correlations can cause underestimates of systemic risk [19].

- We often design higher-level components that tolerate lowerlevel failures, or that convert total failures at a lower level to degraded service at a higher level. Such fault-masking techniques can yield higher overall availability than simple math suggests, reducing the utility of trying to improve component SLOs.

These sub-system SLO choices also require balancing costs; it might be much cheaper to improve some components vs. others, or it might be necessary to re-architect the entire system 
instead of throwing resources at bullet-proofing a particular dependency.

\subsection{Tactical vs. strategic SLOs}

We use SLOs for both tactical (operational) decisions should pagers go off? Do we need to fix something now? - and strategic (compositional) decisions - what can the designers of client systems rely on?

Tactical SLOs, to be useful, must be easily and cheaply measured on short timescales, and must make sense to service operators, but do not need to be sensible for customers. E.g., "packet loss rate" could be a tactical SLO; it is easy to measure, and early operator intervention can avoid a strategic-SLO (user-visible) failure. (Note that tactical SLOs can also be used in automatic control loops, not just for operator interventions.)

Strategic SLOs must be stated in terms that a client system's designer can understand and build on, but can often be stated and measured over relatively long timescales. If these are not high-nines SLOs, that is OK; the client designers at least know they must compensate for that.

It is tempting to use one SLO for both tactical and strategic purposes, but we believe that leads to trouble; end-to-end composition of systems from components is not the same problem as managing the operation of each component. The metrics and timescales for tactical and strategic SLOs often could and should be different.

In another paper in this workshop, Huang et al. point out that mechanisms for checking compliance with tactical SLOs can suffer from "gray failure," where subtle faults in the infrastructure go un-detected by the provider's failure detectors, but still cause outages to end-user applications [23]. They discuss this problem of "differential observability" and propose some possible approaches to ameliorating it.

\section{Availability is like security ... somewhat}

We have explained why defining availability SLOs can be difficult. In this section, we argue that one should think about availability with the same kind of mindset that we have learned to use when thinking about security [36]. Of course availability is not exactly the same as security - but it is also not exactly the same as fault tolerance, either. This analogy to security has helped us clarify our thinking about availability.

Many aspects of the analogy reflect the need, in both domains, for "adversarial thinking" on the part of system designers and operators:

- When designing a system for either security or availability, one should start with a well-defined threat model [42]. Both kinds of "threats" include misconfigurations, unknown dependencies, poor assumptions, and system overload.

- In a system that actually approaches 6 nines of availability, almost every loss-of-availability failure is a new one (e.g., over 2 years, Google's networks experienced at least 103 previously unknown outages [21]). Therefore, these are hard to anticipate, analogous to "zero-day" security vulnerabilities.

- Measuring past availability of 6 nines cannot tell you whether you'll get the same in the future, in the same way that measuring past security compromises cannot tell you whether you are immune from future ones.

Other aspects of the analogy relate to mitigation:
- Configuration errors afflict both security [31] and availability [21], especially because configurations are often updated by operators who have less understanding of a system's design than its developers, and who are under time pressure. The overall system needs to tolerate misconfiguration, not merely detect it (although detecting misconfigurations is certainly worth the effort); designs that make it hard to express a misconfiguration are even better.

- A service can be designed to "fail-static": to continue to operate when another service, on which it depends, has failed. (We discuss this more in $\S 6$.) Fail-static for availability is analogous to default-deny [36] in security mechanisms. Both follow the principle of "if you don't know what to do, do the least harmful thing."

- Systems need to detect and mitigate large-scale, fastspreading failures, both for availability and security, even before you know what caused them. Security practices can involve locking down a system during crisis (e.g., disconnecting it from the Internet, or disabling changes to the userauthentication database). Similar availability-preserving practices include mechanisms to rapidly, sometimes automatically, roll back to a known-good configuration [41] or to implement a "Big Red Button" [21] that disconnects a complex, new part of a system from the simpler, older part.

The analogy can also guide system-wide choices:

- Security and availability both conflict with execution velocity. In both cases, we have to discourage or prevent user/developer/operator short-cuts, which become sources of risk. Also, we avoid new-feature introductions into a system whose availability has been poor.

- Practical solutions require defense in depth, rather than relying only on proof-based and test-based confidence in the system's behavior. No one mechanism guarantees overall availability, in the same way that no one mechanism provides full security.

The analogy is not perfect: availability is not like security in many ways. For example, redundancy is far more useful in preventing availability problems than security problems, and while attackers have incentives and skills to seek out new exploits, many causes of unavailability (aside from DoS attacks) are benign. But when a design or implementation flaw takes a provider's infrastructure offline, customers might not care whether the proximate cause was malice or just bad luck.

\subsection{Analogous operational practices}

Availability, like security, also requires adherence to good operational practices, which cannot be implemented entirely by added or improved software. Instances abound where serious security breaches have been attributed to poor organizational practices, for example [35] and [37].

Reviews: Good security begins with early-stage design reviews by outside experts. These reviews require consultation with security experts, rather than relying on the limited security-related experience of the average engineer. Similarly, availability-conscious organizations should use an expertconsultant process for early-stage availability reviews. 
Penetration testing: In a "penetration test," a team of security experts, distinct from the system designers and operators, attempts to defeat or evade the system's security mechanisms. System designers cannot be expected to fully understand either their security-threat or availability-threat models, or to test whether their designs properly defend against these threats. Our Site Reliability Engineers regularly play a "Wheel of Misfortune" game to role-play human responses to possible disasters [5, Ch. 28]. Regular testing of disaster recovery mechanisms is critical to ensure they will actually work when needed.

\section{Past research: successes and gaps}

Fifty years of active research into networked-systems availability has had only limited impact on engineering practice. Why? We posit that the most influential outcomes from that body of research have largely been point solutions that are either domain-specific or address just one aspect of availability.

For example, Byzantine Fault Tolerance (BFT) protocols [2, $11,14,28,45]$ are not widely deployed. This could be due to performance and scaling concerns, or the perceived risk to overall availability associated with running a synchronous protocol in the data center [6]. As a real-world example, we had a router within the network in Fig. 1 that falsely reported a lightlyloaded link as $100 \%$ utilized. Control-plane services faithfully reported this "alternative fact" to BwE, which tried to throttle server traffic until the reported link utilization dropped - which it never did. The network risked becoming unavailable to some servers, even though all of the services were fully "available." While this is a form of Bzyantine failure, $3 f+1$ replication of routers is infeasible; we must instead engineer the control plane to detect anomalous inputs. Critically, while we can quantify the risk of Byzantine faults, we lack a framework for systematically trading off that risk against availability and performance guarantees.

Providers have not entirely ignored research results. Paxos [30] is widely used within Google [4, 8] and elsewhere [24]. Many academics and practitioners have explored the tradeoffs between consistency, performance and availability $[1,9,18,43,46]$, efforts that have had tangible impact on global-scale production services.

The notion of performability [32] captures, via a Markov model, how the performance of a multi-node hardware or software system degrades as a function of the availability of individual nodes. To model availability, we would like to have the dual of performability: a formal way to express how availability varies as a function of the performance of the nodes.

Several authors have provided useful taxonomies. Jain provides a taxonomy of performance evaluation metrics [25] in which availability is as important as speed and reliability. Sterbenz et al. offer an architectural framework for network resilience [40], identifying classes of engineering solutions to operational threats, and metrics to assess effectiveness of these techniques. However, we are not aware of widespread adoption of either approach; industry mostly seems to treat performance and availability as unrelated issues.

\section{Techniques for improving availability}

Here we discuss a few techniques to address end-to-end infrastructure availability, which are distinct from prior pointsolutions research and the fundamental technique of redundancy. A precise and principled definition of availability for the target system is, of course, a prerequisite.

Don't try to solve availability in one layer: While an IaaS provider cannot expect its tenants to tolerate high failure rates, it can work cooperatively with them to achieve higher redundancy at lower cost than it could provide unilaterally. Specifically, providers encourage tenants to replicate their services across VMs in different zones or regions.

Such cooperation allows a provider to engineer single zones for somewhat lower availability (therefore lower cost) than a tenant requires overall; this is analogous to defense-in-depth. However, any services that are inherently global, such as account creation and authentication, or BwE in Fig. 1, must meet higher availability targets. Providers therefore need to be cautious about introducing dependencies on too many global-scope services.

Fail-static: Govindan et al. discuss how a network's realtime packet-forwarding layer can keep operating even when its SDN controller crashes and restarts ${ }^{3}$; this is analogous to default-deny. We can also apply fail-static to service stacks, between multiple layers. For example, in Fig. 1, BwE can stash a recent model from the current-model service, to use if the latter service fails. During such a failure, BwE's view of the network will grow stale, as links fail or recover, so its throttling might need to gradually become more conservative, but it can still respond to new demands. 4

Generalizing fail-static poses challenges: reliably detecting component failure, designing a system to continue to operate in such cases, testing against hard-to-anticipate failures, and estimating overall system availability with fail-static enabled for sub-components. Availability is no longer as simple as multiplying failure probabilities.

Fail-static also requires retraining developers. Instead of writing fail-stop code using ASSERT and PANIC, they must reason about unwinding gracefully after errors.

Avoid cyclic dependencies: Large organizations that build complex distributed systems tend to accidentally introduce dependency cycles, which can create unexpected correlations between apparently independent systems, and can make it extremely hard to recover when all instances of a component have failed. Or, if a failure detector depends on a system that has failed, failures can go unnoticed (and similarly for access to logs necessary to understand the cause of a failure [21]). Cycles can be detected if the global graph is known, but developers often have only a local and informal understanding of the dependency graph; requiring explicit registrations of inter-component dependencies can help build such a graph. However, some cycles are hard to live without.

We lack space for details on these other techniques:

- Replicated systems suffer when replica failures are correlated; pushing the same configuration change to all replicas at once creates a correlation, so config-push policies and mechanism should exploit "canarying" and gradual rollout [21].

\footnotetext{
3 Their term is "fail-open," but we prefer "fail-static."

${ }^{4}$ This is similar to "hoarding" for disconnected computing [27].
} 
- Emergency systems must be tested before actual emergencies; if the system design can execute its "emergency" code paths even in normal operation, at least occasionally, recovery times tend to improve [5, Ch. 17].

- Capacity planning, to avoid "outages" caused by predictable increases in demand that would lead to overloading even nonfailed resources.

- Partitioning a larger system into $N$ distinct scopes ("colors") with almost no sharing, means that faults (especially misconfigurations and maintenance errors) remove only $1 / N$ capacity [21].

- Continuous assessment of risk of failures due to faults that reduce redundancy without yet causing capacity loss; redundant systems are hard to probe for risk from outside, unless designed to support this [21,38]. This includes monitoring implementation-internal metrics, such as queue lengths, memory consumption, and RPC delays, to detect if the system is at risk of reaching internal limits. (E.g., hard-coded timeout values can be an implicit threat to availability, unless response times are monitored w.r.t. this risk.)

\subsection{Availability is not just avoiding failure}

Availability suffers when Mean Time Between Failures (MTBF) is too low, but also when Mean Time To Repair (MTTR) is too high. Govindan et al. [21] report MTTRs of 10s100 s of minutes, so one such failure per year limits availability to no better than 4 nines. Overall availability might be better served by improving MTTR, together with rapid and accurate failure detection [13], rather than by expensive approaches to reducing $\mathrm{MTBF}$.

This point is recognized by some past work that addresses MTTR (e.g., Recovery Oriented Computing [34] and Microreboot [10]). Nevertheless, in a recent high-profile incident, code in Cloudflare's "RRDNS" DNS proxy saw time go backward as a result of the leap second in December 2017, starting a chain of events that caused "some DNS resolutions to some Cloudflare managed web properties" to fail. While the original non-monotonic time anomaly lasted just one second, it took 90 minutes to restore DNS service [22]. (This also illustrates the need to define unusual SLOs in order to support composition; here, the DNS proxy needs to cope with a time-service SLO in which time might go backwards, but not by much, and not often.)

\section{Next Steps}

We have explained why defining and measuring availability is difficult, and we have described a number of techniques for improving availability. We identify a way to think about availability that draws from how we think about security, analogous to Saltzer \& Schroeder on security [36]. We hope this approach leads to a general framework that provides principles and mechanisms for:

- Clearly defining multi-dimensional SLIs and their composition into system-level SLOs, and then choosing sub-system SLOs.

- Defining sub-system SLOs to minimize overall system cost while attaining end-to-end SLOs, rather than focusing on point solutions that try to provide "perfect" availability for just one layer.
- Given a system or component SLO, predicting whether the system's design will actually meet that SLO, especially given complex dependency graphs, and partial or cascading failures. (Keeton et al. applied this approach to storage systems [26]).

- Deciding when to use design patterns such as fail-static vs. fail-stop, and how best to use them.

Acknowledgments: We thank our colleagues Martín Abadi, Yaniv Aknin, Emilie Danna, Dina Papagiannaki, John Reese, David Wetherall, and John Wilkes for their help. The anonymous reviewers made wonderful suggestions; we wish we had space to simply include their reviews verbatim.

\section{References}

[1] Daniel J. Abadi. Consistency Tradeoffs in Modern Distributed Database System Design. Computer, pages 37-42, February 2012.

[2] Michael Abd-El-Malek, Gregory R. Ganger, Garth R. Goodson, Michael K. Reiter, and Jay J. Wylie. Fault-Scalable Byzantine Fault-Tolerant Services. In Proc. SOSP. ACM, 2005.

[3] Amazon. Summary of the Amazon DynamoDB Service Disruption and Related Impacts in the US-East Region. https://aws.amazon.com/message/5467D2/, September 2015. Last accessed on Jan 12, 2017.

[4] Jason Baker, Chris Bond, James C. Corbett, JJ Furman, Andrey Khorlin, James Larson, Jean-Michel Leon, Yawei Li, Alexander Lloyd, and Vadim Yushprakh. Megastore: Providing Scalable, Highly Available Storage for Interactive Services. In Proceedings of the Conference on Innovative Data system Research (CIDR), pages 223-234, 2011.

[5] Betsy Beyer, Chris Jones, Jennifer Petoff, and Niall Richard Murphy, editors. Site Reliability Engineering. O'Reilly Media, Inc., 2016.

[6] Ken Birman, Gregory Chockler, and Robbert van Renesse. Toward a cloud computing research agenda. SIGACT News, 40:68-80, June 2009.

[7] Eric Brewer. CAP Twelve Years Later: How the "Rules" Have Changed. Computer, 45(2):23-29, Feb 2012.

[8] Mike Burrows. The Chubby lock service for loosely-coupled distributed systems. In Proc. OSDI, pages 335-350, 2006.

[9] Brad Calder, Ju Wang, Aaron Ogus, Niranjan Nilakantan, Arild Skjolsvold, Sam McKelvie, Yikang Xu, Shashwat Srivastav, Jiesheng Wu, Huseyin Simitci, Jaidev Haridas, Chakravarthy Uddaraju, Hemal Khatri, Andrew Edwards, Vaman Bedekar, Shane Mainali, Rafay Abbasi, Arpit Agarwal, Mian Fahim ul Haq, Muhammad Ikram ul Haq, Deepali Bhardwaj, Sowmya Dayanand, Anitha Adusumilli, Marvin McNett, Sriram Sankaran, Kavitha Manivannan, and Leonidas Rigas. Windows Azure Storage: A Highly Available Cloud Storage Service with Strong Consistency. In Proc. SOSP. ACM, 2011.

[10] George Candea, Shinichi Kawamoto, Yuichi Fujiki, Greg Friedman, and Armando Fox. Microreboot - A Technique for Cheap Recovery. In Proc. OSDI, 2004.

[11] Miguel Castro and Barbara Liskov. Practical Byzantine Fault Tolerance and Proactive Recovery. Transactions on Computer Systems (TOCS), 20:398461, November 2002.

[12] HealthCare.gov Progress and Performance Report. Centers for Medicare \& Medicaid Services, https://www.cms.gov/newsroom/mediareleasedatabase/pressreleases/2013-press-releases-items/2013-12-01.html.

[13] Mike Y. Chen, Emre Kiciman, Eugene Fratkin, Armando Fox, and Eric Brewer. Pinpoint: Problem Determination in Large, Dynamic Internet Services. In Proc. DSN, pages 595-604, 2002.

[14] Allen Clement, Edmund Wong, Lorenzo Alvisi, and Mike Dahlin. Making Byzantine Fault Tolerant Systems Tolerate Byzantine Faults. In Proc. NSDI. USENIX, 2009.

[15] Michael Conley, Amin Vahdat, and George Porter. Achieving Cost-efficient, Data-intensive Computing in the Cloud. In Proc. SoCC, pages 302-314, 2015.

[16] James C. Corbett, Jeffrey Dean, Michael Epstein, Andrew Fikes, Christopher Frost, J. J. Furman, Sanjay Ghemawat, Andrey Gubarev, Christopher Heiser, Peter Hochschild, Wilson Hsieh, Sebastian Kanthak, Eugene Kogan, Hongyi Li, Alexander Lloyd, Sergey Melnik, David Mwaura, David Nagle, Sean Quinlan, Rajesh Rao, Lindsay Rolig, Yasushi Saito, Michal Szymaniak, Christopher Taylor, Ruth Wang, and Dale Woodford. Spanner: Google's Globally Distributed Database. ACM Trans. Comput. Syst., 31(3):8:1-8:22, August 2013.

[17] Jeffrey Dean and Luiz André Barroso. The Tail at Scale. CACM, 56(2):7480, February 2013.

[18] Giuseppe DeCandia, Deniz Hastorun, Madan Jampani, Gunavardhan Kakulapati, Avinash Lakshman, Alex Pilchin, Swaminathan Sivasubramanian, Peter Vosshall, and Werner Vogels. Dynamo: Amazon's Highly Available Key-value Store. In Proc. SOSP. ACM, 2007. 
[19] Bryan Ford. Icebergs in the Clouds: the Other Risks of Cloud Computing. In Proc. HotCloud, 2012.

[20] Adam Glick, Jason Roth, Ralph Squillace, and Simon Rolfe. Disaster recovery and high availability for applications built on $\mathrm{Mi}$ crosoft Azure. https://docs.microsoft.com/en-us/azure/resiliency/resiliencydisaster-recovery-high-availability-azure-applications, August 2016. Last accessed on Jan 12, 2017.

[21] Ramesh Govindan, Ina Minei, Mahesh Kallahalla, Bikash Koley, and Amin Vahdat. Evolve or Die: High-Availability Design Principles Drawn from Google's Network Infrastructure. In Proc. SIGCOMM, pages 58-72, 2016.

[22] John Graham-Cumming. How and why the leap second affected Cloudflare DNS. https://blog.cloudflare.com/how-and-why-the-leap-second-affectedcloudflare-dns/, January 2017.

[23] Peng Huang, Chuanxiong Guo, Lidong Zhou, Jacob R. Lorch, Yingnong Dang, Murali Chintalapati, and Randolph Yao. Gray failure: The achillesâĂŹ heel of cloud-scale systems. In Proc. HotOS, May 2017.

[24] Patrick Hunt, Mahadev Konar, Flavio P. Junqueira, and Benjamin Reed. ZooKeeper: Wait-free Coordination for Internet-scale Systems. In Proc. USENIX ATC, 2010

[25] Raj Jain. The Art of Computer Systems Performance Analysis, chapter 3. John Wiley, 1991.

[26] Kimberley Keeton, Cipriano Santos, Dirk Beyer, Jeffrey Chase, and John Wilkes. Designing for Disasters. In Proc. FAST, pages 59-62, 2004.

[27] James J. Kistler and M. Satyanarayanan. Disconnected Operation in the Coda File System. Trans. Comput. Syst., 10(1):3-25, February 1992

[28] Ramakrishna Kotla, Lorenzo Alvisi, Mike Dahlin, Allen Clement, and Edmund Wong. Zyzzyva: Speculative Byzantine Fault Tolerance. In Proc. SOSP. ACM, 2007.

[29] Alok Kumar, Sushant Jain, Uday Naik, Anand Raghuraman, Nikhil Kasinadhuni, Enrique Cauich Zermeno, C. Stephen Gunn, Jing Ai, Björn Carlin, Mihai Amarandei-Stavila, Mathieu Robin, Aspi Siganporia, Stephen Stuart, and Amin Vahdat. BwE: Flexible, Hierarchical Bandwidth Allocation for WAN Distributed Computing. In Proc. SIGCOMM '15, 2015.

[30] Leslie Lamport. The part-time parliament. Transactions on Computer Systems (TOCS), 16:133-169, May 1998

[31] Butler W. Lampson. Computer Security in the Real World. Computer, 37(6):37-46, June 2004

[32] John F. Meyer. Performability: a retrospective and some pointers to the future. Performance Evaluation, 14:139-156, 1992.

[33] Robinson Meyer. The Secret Startup That Saved the Worst Website in America. The Atlantic, July 2015.

[34] David Patterson, Aaron Brown, Pete Broadwell, George Candea, Mike Chen, James Cutler, Patricia Enriquez, Armando Fox, Emre KÄścÄśman, Matthew Merzbacher, David Oppenheimer, Naveen Sastry, William Tetzlaff, Jonathan Traupman, and Noah Treuhaft. Recovery Oriented Computing (ROC): Motivation, Definition, Techniques, and Case Studies. Technical Report UCB//CSD-02-1175, U.C. Berkeley Computer Science, March 2002.

[35] Teri Radichel. Case Study: Critical Controls that Could Have Prevented Target Breach. SANS Institute InfoSec Reading Room, August 2014

[36] Jerome H. Saltzer and Michael D. Schroeder. The Protection of Information in Computer Systems. Proc. IEEE, 63(9):1278-1308, September 1975.

[37] Gabriel Sanchez. Case Study: Critical Controls that Sony Should Have Implemented. SANS Institute InfoSec Reading Room, June 2015.

[38] Mehul A. Shah, Mary Baker, Jeffrey C. Mogul, and Ram Swaminathan. Auditing to Keep Online Storage Services Honest. In Proc. HOTOS, pages 11:1-11:6, 2007.

[39] Arjun Singh, Joon Ong, Amit Agarwal, Glen Anderson, Ashby Armistead, Roy Bannon, Seb Boving, Gaurav Desai, Bob Felderman, Paulie Germano, Anand Kanagala, Jeff Provost, Jason Simmons, Eiichi Tanda, Jim Wanderer, Urs Hölzle, Stephen Stuart, and Amin Vahdat. Jupiter Rising: A Decade of Clos Topologies and Centralized Control in Google's Datacenter Network. In Proc. SIGCOMM, pages 183-197, 2015.

[40] James P. G. Sterbenz, David Hutchison, Egemen K. Çetinkaya, Abdul Jabbar, Justin P. Rohrer, Marcus Schöller, and Paul Smith. Resilience and survivability in communication networks: Strategies, principles, and survey of disciplines. Computer Networks, 54:1245-1265, 2010.

[41] Yu-Wei Eric Sung, Xiaozheng Tie, Starsky H.Y. Wong, and Hongyi Zeng. Robotron: Top-down Network Management at Facebook Scale. In Proc. SIGCOMM, pages 426-439, 2016.

[42] Frank Swiderski and Window Snyder. Threat Modeling. Microsoft Press, Redmond, WA, USA, 2004

[43] Douglas B. Terry, Vijayan Prabhakaran, Ramakrishna Kotla, Mahesh Balakrishnan, Marcos K. Aguilera, and Hussam Abu-Libdeh. Consistencybased Service Level Agreements for Cloud Storage. In Proc. SOSP. ACM, 2013.

[44] John Wilkes. Utility Functions, Prices, and Negotiation. In Rajkumar Buyya and Kris Bubendorfer, editors, Market-Oriented Grid and Utility Computing, Wiley Series on Parallel and Distributed Computing, chapter 4, pages 67-88. John Wiley \& Sons, Hoboken, NJ, October 2009.
[45] Jian Yin, Jean-Philippe Martin, Arun Venkataramani, Lorenzo Alvisi, and Mike Dahlin. Separating Agreement from Execution for Byzantine Fault Tolerant Services. In Proc. SOSP. ACM, 2003.

[46] Haifeng Yu and Amin Vahdat. Design and evaluation of a continuous consistency model for replicated services. In Proc. OSDI, 2000. 